\title{
On upper transversals in 3-uniform hypergraphs
}

\author{
Michael A. Henning ${ }^{1, *}$ Anders Yeo ${ }^{1,2}$ \\ ${ }^{1}$ Department of Pure and Applied Mathematics \\ University of Johannesburg \\ Auckland Park, 2006 South Africa \\ mahenning@uj.ac.za \\ ${ }^{2}$ Department of Mathematics and Computer Science \\ University of Southern Denmark \\ Campusvej 55, 5230 Odense M, Denmark \\ andersyeo@gmail.com
}

Submitted: Aug 29, 2017; Accepted: Oct 16, 2018; Published: Nov 2, 2018

(C) The authors. Released under the CC BY-ND license (International 4.0).

\begin{abstract}
A set $S$ of vertices in a hypergraph $H$ is a transversal if it has a nonempty intersection with every edge of $H$. The upper transversal number $\Upsilon(H)$ of $H$ is the maximum cardinality of a minimal transversal in $H$. We show that if $H$ is a connected 3-uniform hypergraph of order $n$, then $\Upsilon(H)>1.4855 \sqrt[3]{n}-2$. For $n$ sufficiently large, we construct infinitely many connected 3 -uniform hypergraphs, $H$, of order $n$ satisfying $\Upsilon(H)<2.5199 \sqrt[3]{n}$. We conjecture that $\sup _{n \rightarrow \infty}\left(\inf \frac{\Upsilon(H)}{\sqrt[3]{n}}\right)=\sqrt[3]{16}$, where the infimum is taken over all connected 3-uniform hypergraphs $H$ of order $n$.
\end{abstract}

Mathematics Subject Classifications: 05C88, 05C89

\section{Introduction}

In this paper, we continue the study of transversals in hypergraphs. Hypergraphs are systems of sets which are conceived as natural extensions of graphs. A hypergraph $H=$ $(V(H), E(H))$ is a finite set $V(H)$ of elements, called vertices, together with a finite multiset $E(H)$ of nonempty subsets of $V(H)$, called hyperedges or simply edges. A $k$-edge in $H$ is an edge of size $k$. The hypergraph $H$ is $k$-uniform if every edge of $H$ is a $k$-edge. Every 2-uniform hypergraph is a graph. Thus graphs are special hypergraphs. The degree of a vertex $v$ in $H$, denoted by $d_{H}(v)$, is the number of edges of $H$ which contain $v$. The

\footnotetext{
${ }^{*}$ Research supported in part by the South African National Research Foundation and the University of Johannesburg
} 
minimum and maximum degrees among the vertices of $H$ is denoted by $\delta(H)$ and $\Delta(H)$, respectively.

A subset $T$ of vertices in a hypergraph $H$ is a transversal (also called hitting set or vertex cover or blocking set in many papers) if $T$ has a nonempty intersection with every edge of $H$. A vertex hits or covers an edge if it belongs to that edge. The transversal number $\tau(H)$ of $H$ is the minimum size of a transversal in $H$, while the upper transversal number $\Upsilon(H)$ of $H$ is the maximum cardinality of a minimal transversal in $H$. In hypergraph theory the concept of transversal is fundamental and well studied. The major monograph [1] of hypergraph theory gives a detailed introduction to this topic. Transversals in hypergraphs are well studied in the literature (see, for example, $[3,4,11,12,13]$ for recent results and further references).

A set $S$ of vertices in a graph $G$ is a dominating set of $G$ if each vertex in $V(G) \backslash S$ has a neighbor in $S$. A set is independent if no two vertices in it are adjacent. An independent dominating set of $G$ is a set that is both dominating and independent in $G$. The independent domination number of $G$, denoted by $i(G)$, is the minimum cardinality of an independent dominating set. Domination is well studied in graph theory and we refer the reader to the monographs $[9,10]$ which detail and survey many results on the topic. A survey of known results on independent domination in graphs can be found in [8].

\section{$2 \quad$ Main Results}

We have two immediate aims in this paper. First to provide a sharp lower bound on the upper transversal number of graphs. Secondly to present a lower bound on the upper transversal number of 3-uniform hypergraphs, and to show that this bound is a sense asymptotically best possible. More precisely, we prove the following results, where we use the notation $n_{H}=|V(H)|$ to denote the order of $H$. Proofs of Theorem 1 and Theorem 2 are given in Section 3 and Section 4, respectively.

Theorem 1. If $H$ is a connected graph with $\delta(H) \geqslant \delta$, then

$$
\Upsilon(H) \geqslant 2 \sqrt{\delta n_{H}}-2 \delta,
$$

and this bound is sharp.

Theorem 2. If $H$ is a connected 3-uniform hypergraph, then

$$
\Upsilon(H)>\sqrt[3]{\frac{n_{H}}{0.305}}-2>1.4855 \sqrt[3]{n_{H}}-2 .
$$

Further, there exist infinitely many connected 3-uniform hypergraphs $H$ of sufficiently large order $n_{H}$ satisfying

$$
\Upsilon(H)<\sqrt[3]{16 \cdot n_{H}}<2.52 \sqrt[3]{n_{H}}
$$




\section{Proof of Theorem 1}

Recall that a transversal in a graph is a set of vertices covering all the edges of the graph, where a vertex covers an edge if it is incident with it. Theorem 1 can be restated as follows.

Theorem 1. If $G$ is a connected graph of order $n$ with $\delta(G) \geqslant \delta$, then $\Upsilon(G) \geqslant 2 \sqrt{\delta n}-2 \delta$, and this bound is sharp.

In order to prove Theorem 1, we first establish a relationship between the upper transversal number and independent domination number of a graph.

Theorem 3. If $G$ is an isolate-free graph on $n$ vertices, then $i(G)+\Upsilon(G)=n$.

Proof. Let $G$ be an isolate-free graph. Let $S$ be an independent dominating set in $G$ of minimum cardinality, and so $|S|=i(G)$. Let $T=V(G) \backslash S$ and note that $T$ is a transversal in $G$ as $S$ is an independent set. Since every vertex in $T$ has a neighbor in $S$, we furthermore note that $T$ is a minimal transversal, which implies that $\Upsilon(G) \geqslant|T|=$ $n-|S|=n-i(G)$.

Conversely, let $T$ be a minimal transversal in $G$ of maximum cardinality, and so $|T|=\Upsilon(G)$. Let $S=V(G) \backslash T$ and note that $S$ is an independent set as $T$ is a transversal. Since $T$ is a minimal transversal, every vertex in $T$ has a neighbor in $S$, implying that $S$ is an independent dominating set in $G$. Therefore, $i(G) \leqslant|S|=n-|T|=n-\Upsilon(G)$. Consequently, $i(G)+\Upsilon(G)=n$.

Favaron [5] was the first to prove the following upper bound on the independent domination of a graph with no isolated vertex: If $G$ is an isolate-free graph of order $n$, then $i(G) \leqslant n+2-2 \sqrt{n}$. We remark that this result also follows from a result due to Bollobás and Cockayne [2] (and was also proved in [6]). Sun and Wang [14] proved the following more general result, which was originally posed as a conjecture by Favaron [5] and was proved for $\delta=2$ by Glebov and Kostochka [7].

Theorem 4. ([14]) If $G$ is a graph of order $n$ with $\delta(G) \geqslant \delta$, then $i(G) \leqslant n+2 \delta-2 \sqrt{\delta n}$.

Theorem 1 is an immediate consequence of Theorem 3 and Theorem 4. That this bound is sharp, follows from a result of Favaron [5] who showed that for every positive integer $\delta$, the bound in Theorem 4 is attained for infinitely many graphs. The same graphs achieve equality in the bound for Theorem 1 . For example, for $c \geqslant 2$, let $G_{c}$ be the connected graph constructed as follows. Let $F_{c}$ be the complete graph of order $c$, and so $F_{c} \cong K_{c}$. For every vertex $v$ of $F_{c}$, add $c-1$ new vertices $v_{1}, \ldots, v_{c-1}$ and add the $c-1$ edges $v v_{i}$ for all $i \in[c-1]$. Let $G=G_{c}$ denote the resulting graph of order $n=c^{2}$. We note that all the new vertices added to $F_{c}$ have degree 1 in $G_{c}$. Every transversal in $G_{c}$ must contain all except possibly one vertex of $F_{c}$ in order to cover all the edges of $F_{c}$. If a minimal transversal in $G_{c}$ contains exactly $c-1$ vertices of $F_{c}$, say all vertices of $F_{c}$ except for $v$, then the transversal contains exactly $c-1$ vertices not in $F_{c}$, namely $v_{1}, \ldots, v_{c-1}$, in order to cover the edges $v v_{i}$ for all $i \in[c-1]$. Such a minimal transversal therefore has 
size exactly $2(c-1)$. If a minimal transversal in $G_{c}$ contains all $c$ vertices of $F_{c}$, then it contains no other vertex of $G_{c}$ and therefore has size exactly $c$. Therefore, the connected graph $G$ of order $n=c^{2}$ satisfies $\Upsilon(G)=\max \{2(c-1), c\}=2(c-1)=2 \sqrt{n}-2$, noting that $c \geqslant 2$. Thus, the bound of Theorem 1 when $\delta=1$ is sharp. For every $\delta \geqslant 2$ one can similarly show that Theorem 1 is tight.

As a special case of Theorem 1 , if $H$ is a connected 2-uniform hypergraph of order $n \geqslant$ 2 , then $\Upsilon(H) \geqslant 2 \sqrt{n}-2$. When $n \geqslant 3$, we observe that $2 \sqrt{n}-2 \geqslant \sqrt{\frac{1}{2} n}$. Further, we observe that when $n=2, \Upsilon(H)=1=\sqrt{\frac{1}{2} n}$. Thus, as an immediate consequence of Theorem 1 we observe that if $H$ is a connected graph, then $\Upsilon(H) \geqslant \sqrt{\frac{1}{2} n_{H}}$.

\section{Proof of Theorem 2}

We first prove the lower bound in Theorem 2 .

Theorem 5. If $H$ is a connected 3-uniform hypergraph, then $\Upsilon(H)>\sqrt[3]{\frac{n_{H}}{0.305}}-2$.

Proof. Let $H$ be a connected 3-uniform hypergraph of order $n_{H}$ and let $T$ be a minimal transversal of maximum size. Let $T=\left\{t_{1}, t_{2}, \ldots, t_{c}\right\}$, and so $\Upsilon(H)=|T|=c$. For all $i$ and $j$ where $1 \leqslant i<j \leqslant c$ and for all $k \in[c]$, define $Z_{i, j}, E_{k}$ and $Y_{k}$ as follows.

$$
\begin{aligned}
Z_{i, j} & =\left\{v \in V(H) \backslash T \mid\left\{t_{i}, t_{j}, v\right\} \in E(H)\right\} \\
E_{k} & =\left\{e \in E(H) \mid V(e) \cap T=\left\{t_{k}\right\}\right\} \\
Y_{k} & =V\left(E_{k}\right) \backslash\left\{t_{k}\right\} .
\end{aligned}
$$

We note that $Y_{k} \subseteq V(H) \backslash T$ for each $k \in[c]$. Let $Q \subseteq V(T)$ be a minimum set of vertices in $T$ that covers all edges that are completely within $T$ (i.e., all edges $e$ with $V(e) \subseteq V(T))$. Possibly, $Q=\emptyset$. Let $q=|Q|$. Renaming vertices of $T$ if necessary, we may assume that $Q=\left\{t_{1}, \ldots, t_{q}\right\}$. Let

$$
\begin{aligned}
\mathcal{I} & =\{(i, j) \mid 1 \leqslant i<j \leqslant c\} \\
\mathcal{I}_{q} & =\{(i, j) \mid 1 \leqslant i \leqslant q \text { and } i<j \leqslant c\} \\
\mathcal{I}_{>q} & =\{(i, j) \mid q+1 \leqslant i<j \leqslant c\} .
\end{aligned}
$$

We note that $\mathcal{I}=\mathcal{I}_{q} \cup \mathcal{I}_{>q}$. We proceed further with the following claims.

Claim 6. $\left|Z_{i, j}\right| \leqslant c-q$ for all $(i, j) \in \mathcal{I}$.

Proof. Suppose, to the contrary, that $\left|Z_{i, j}\right|>c-q$ for some $i$ and $j$, where $1 \leqslant i<j \leqslant c$. Let $R=V(H) \backslash\left\{t_{i}, t_{j}\right\}$. Clearly, $R$ is a transversal in $H$ as it contains all vertices in $H$ except two and $H$ is 3-uniform. Let $R^{\prime}$ be obtained from $R$ by removing vertices until we get a minimal transversal in $H$. We note that $Z_{i, j} \subseteq R^{\prime}$ since each vertex $z \in Z_{i, j}$ is needed in order to cover the edge $\left\{t_{i}, t_{j}, z\right\}$. Further, $R^{\prime}$ contains at least $q$ vertices from $T$ in order to cover the edges that are contained entirely within $T$. Hence, $\Upsilon(H) \geqslant\left|R^{\prime}\right| \geqslant\left|Z_{i, j}\right|+q>c$, contradicting the fact that $\Upsilon(H)=c$. 
Claim 7. $\left|\bigcup_{(i, j) \in \mathcal{I}_{>q}} Z_{i, j}\right| \leqslant c-q$.

Proof. Suppose, to the contrary, that

$$
\left|\bigcup_{(i, j) \in \mathcal{I}_{>q}} Z_{i, j}\right|>c-q
$$

Let $R=V(H) \backslash\left\{t_{q+1}, \ldots, t_{c}\right\}$. By definition of the set $Q$, every edge of $H$ intersects $\left\{t_{q+1}, \ldots, t_{c}\right\}$ in at most two vertices, implying that $R$ is a transversal in $H$. Let $R^{\prime}$ be obtained from $R$ by removing vertices from $R$ until we get a minimal transversal in $H$. We note that

$$
\bigcup_{(i, j) \in \mathcal{I}_{>q}} Z_{i, j} \subseteq R^{\prime}
$$

since each vertex $z \in Z_{i, j}$ where $q+1 \leqslant i<j \leqslant c$ is needed in order to cover the edge $\left\{t_{i}, t_{j}, z\right\}$. Further, $R^{\prime}$ contains at least $q$ vertices from $Q$ in order to cover the edges that are contained entirely within $T$. Hence,

$$
\Upsilon(H) \geqslant\left|R^{\prime}\right| \geqslant\left|\bigcup_{(i, j) \in \mathcal{I}_{>q}} Z_{i, j}\right|+q>c,
$$

contradicting the fact that $\Upsilon(H)=c$.

Claim 8. $\left|\bigcup_{(i, j) \in \mathcal{I}} Z_{i, j}\right| \leqslant\left(\left(\begin{array}{c}c \\ 2\end{array}\right)-\left(\begin{array}{c}c-q \\ 2\end{array}\right)\right)(c-q)+(c-q)$.

Proof. As observed earlier, $\mathcal{I}=\mathcal{I}_{q} \cup \mathcal{I}_{>q}$. By Claim $6,\left|Z_{i, j}\right| \leqslant c-q$ for all $(i, j) \in \mathcal{I}_{q}$. Since there are $\left(\begin{array}{l}c \\ 2\end{array}\right)-\left(\begin{array}{c}c-q \\ 2\end{array}\right)$ pairs $(i, j) \in \mathcal{I}_{q}$ where $1 \leqslant i \leqslant q$ and $i<j \leqslant c$, we note by Claim 6 and Claim 7 that

$$
\left|\bigcup_{(i, j) \in \mathcal{I}} Z_{i, j}\right| \leqslant\left|\bigcup_{(i, j) \in \mathcal{I}_{q}} Z_{i, j}\right|+\left|\bigcup_{(i, j) \in \mathcal{I}_{>q}} Z_{i, j}\right| \leqslant\left(\left(\begin{array}{c}
c \\
2
\end{array}\right)-\left(\begin{array}{c}
c-q \\
2
\end{array}\right)\right)(c-q)+(c-q) .
$$

Claim 9. $\left|Y_{i}\right| \leqslant\left(\frac{c-q}{2}+1\right)^{2}$ for all $i \in[c]$.

Proof. Suppose, to the contrary, that $\left|Y_{i}\right|>((c-q) / 2+1)^{2}$ for some $i \in[c]$. Let $H^{\prime}$ be the graph with vertex set $V\left(H^{\prime}\right)=Y_{i}$ and with edge set $E\left(H^{\prime}\right)=\left\{e \backslash\left\{t_{i}\right\} \mid e \in E_{i}\right\}$. By Theorem 1 , there is a minimal transversal $T^{\prime}$ in $H^{\prime}$, such that $\left|T^{\prime}\right| \geqslant 2\left(\sqrt{\left|Y_{i}\right|}-1\right)$. Let $R^{\prime}=T^{\prime} \cup\left(T \backslash\left\{t_{i}\right\}\right)$ and note that $R^{\prime}$ is a transversal in $H$. Let $R^{\prime \prime}$ be obtained from $R^{\prime}$ by removing vertices from $R^{\prime}$ until we get a minimal transversal in $H$. In order to cover the edges $E_{i}$, we must have $T^{\prime} \subseteq R^{\prime \prime}$, noting that $T^{\prime}$ is a minimal transversal in $H^{\prime}$. Further, $R^{\prime}$ contains at least $q$ vertices from $T \backslash\left\{t_{i}\right\}$ in order to cover the edges that are contained entirely within $T$. Therefore,

$$
\Upsilon(H) \geqslant\left|R^{\prime \prime}\right| \geqslant\left|T^{\prime}\right|+q \geqslant 2\left(\sqrt{\left|Y_{i}\right|}-1\right)+q>2\left(\sqrt{\left(\frac{c-q}{2}+1\right)^{2}}-1\right)+q=c
$$


contradicting the fact that $\Upsilon(H)=c$.

Since $T$ is a transversal in $H$, we note that

$$
V(H)=T \cup\left(\bigcup_{(i, j) \in \mathcal{I}} Z_{i, j}\right) \cup\left(\bigcup_{i=1}^{c} Y_{i}\right) .
$$

Let $\beta$ be defined such that $(c-q)=\beta c$. We note that $0 \leqslant \beta \leqslant 1$. By Equation (1) and by Claim 8 and 9 , we therefore get the following.

$$
\begin{aligned}
n_{H} & \leqslant|T|+\sum_{i, j}\left|Z_{i, j}\right|+\sum_{i}\left|Y_{i}\right| \\
& \leqslant c+\left(\left(\begin{array}{c}
c \\
2
\end{array}\right)-\left(\begin{array}{c}
c-q \\
2
\end{array}\right)\right)(c-q)+(c-q)+c\left(\frac{c-q}{2}+1\right)^{2} \\
& \leqslant c+\left(\frac{c(c-1)}{2}-\frac{\beta c(\beta c-1)}{2}\right) \beta c+\beta c+c\left(\frac{\beta c}{2}+1\right)^{2} \\
& =c+\frac{1}{2}\left(c^{2}-c-\beta^{2} c^{2}+\beta c\right) \beta c+\beta c+c\left(\frac{\beta^{2} c^{2}}{4}+\beta c+1\right) \\
& =c^{3}\left(\frac{\beta}{2}-\frac{\beta^{3}}{2}+\frac{\beta^{2}}{4}\right)+c^{2}\left(\frac{-\beta}{2}+\frac{\beta^{2}}{2}+\beta\right)+c(2+\beta) \\
& =\frac{c^{3}}{4}\left(-2 \beta^{3}+\beta^{2}+2 \beta\right)+c^{2}\left(\frac{\beta}{2}+\frac{\beta^{2}}{2}\right)+c(2+\beta) .
\end{aligned}
$$

Let

$$
f(\beta)=-2 \beta^{3}+\beta^{2}+2 \beta .
$$

The maximum value of $f(\beta)$ when $0 \leqslant \beta \leqslant 1$ is obtained when $\beta=(1+\sqrt{13}) / 6$, noting that $0=f^{\prime}(\beta)=-6 \beta^{2}+2 \beta+2$ implies $\beta=(1 \pm \sqrt{13}) / 6$. Therefore, $f(\beta) \leqslant$ $f\left(\frac{1+\sqrt{13}}{6}\right)<1.22$ for all $0 \leqslant \beta \leqslant 1$, implying by our earlier observations that

$$
\begin{aligned}
n_{H} & \leqslant \frac{c^{3}}{4}\left(-2 \beta^{3}+\beta^{2}+2 \beta\right)+c^{2}\left(\frac{\beta}{2}+\frac{\beta^{2}}{2}\right)+c(2+\beta) \\
& <\frac{c^{3}}{4}(1.22)+c^{2}\left(\frac{1}{2}+\frac{1}{2}\right)+c(2+1) \\
& =0.305 c^{3}+c^{2}+3 c \\
& <0.305(c+2)^{3},
\end{aligned}
$$

and so $\Upsilon(H)=c>\sqrt[3]{\frac{n_{H}}{0.305}}-2$. This completes the proof of Theorem 5 .

We remark that $\sqrt[3]{\frac{1}{0.305}}>1.48559$, and so as a consequence of Theorem 5, if $H$ is a connected 3-uniform hypergraph of order $n \geqslant 3$, then $\Upsilon(H)>1.4855 \sqrt[3]{n}-2$. When $n \geqslant 17$, we observe that $1.4855 \sqrt[3]{n}-2>\sqrt[3]{\frac{1}{3} n}$. Further, we observe that when $n=3$, $\Upsilon(H)=1=\sqrt[3]{\frac{1}{3} n}$, while for $4 \leqslant n \leqslant 16, \Upsilon(H) \geqslant 2>\sqrt[3]{\frac{1}{3} n}$. Thus, as an immediate consequence of Theorem 5, we observe that if $H$ is a connected 3-uniform hypergraph, 
then $\Upsilon(H) \geqslant \sqrt[3]{\frac{n_{H}}{3}}$. We show next that the lower bound in Theorem 5 is asymptotically best possible.

Proposition 10. For all $n \geqslant 3$, there exists a connected 3-uniform hypergraph $H=H_{n}$ of order $n_{H}=\frac{1}{2}\left(n^{3}-n^{2}+2 n\right)$ such that

$$
\Upsilon(H)=\sqrt[3]{16\left(1-\epsilon_{n}\right) \cdot n_{H}} \quad \text { where } \quad \epsilon_{n}=\frac{2 n^{2}-n+1}{n^{3}-n^{2}+2 n} .
$$

Proof. For each $n \geqslant 3$, let $H_{n}$ be the connected 3-uniform hypergraph constructed as follows. Let $F_{n}$ be the complete 3 -uniform hypergraph on $n$ vertices, and so $F_{n}$ has $\left(\begin{array}{l}n \\ 3\end{array}\right)$ hyperedges corresponding to the 3-element subsets of $V\left(F_{n}\right)$. Thus, every set of three vertices in $F_{n}$ belongs to a 3-edge of $F_{n}$. Let $S=V\left(F_{n}\right)$. For every pair of vertices, $\{x, y\}$, in $S$ add $n$ new vertices, $v_{1}^{x y}, v_{2}^{x y}, \ldots, v_{n}^{x y}$ to $F_{n}$ and add the $n$ hyperedges $\left\{x, y, v_{1}^{x y}\right\},\left\{x, y, v_{2}^{x y}\right\}, \ldots,\left\{x, y, v_{n}^{x y}\right\}$. Let $H=H_{n}$ denote the resulting hypergraph of order

$$
n_{H}=\left(\begin{array}{l}
n \\
2
\end{array}\right) n+\left|V\left(F_{n}\right)\right|=\frac{n^{2}(n-1)}{2}+n=\frac{1}{2}\left(n^{3}-n^{2}+2 n\right) .
$$

We note that all the new vertices added to $F_{n}$ have degree 1 in $H_{n}$. Every transversal in $H_{n}$ must contain all $n$ vertices in $F_{n}$, except for possibly two vertices in order to cover all the edges in $F_{n}$. Every minimal transversal in $H_{n}$ contains either exactly $n-1$ vertices of $S$ (and no other vertex in $H_{n}$ ) or exactly $n-2$ vertices of $S$, say all vertices of $S$ except for the vertices $x$ and $y$, and exactly $n$ vertices not in $S$, namely $v_{1}^{x y}, v_{2}^{x y}, \ldots, v_{n}^{x y}$, in order to cover the edges $\left\{x, y, v_{1}^{x y}\right\},\left\{x, y, v_{2}^{x y}\right\}, \ldots,\left\{x, y, v_{n}^{x y}\right\}$, implying that

$$
\Upsilon\left(H_{n}\right)=(|S|-2)+n=2(n-1) .
$$

Therefore, letting $\epsilon_{n}=\frac{2 n^{2}-n+1}{n^{3}-n^{2}+2 n}$, we note that the connected 3-uniform hypergraph $H=H_{n}$ satisfies

$$
\begin{aligned}
\Upsilon(H) & =2(n-1) \\
& =\sqrt[3]{8 \cdot(n-1)^{3}} \\
& =\sqrt[3]{16 \cdot \frac{(n-1)^{3}}{2 n_{H}} \cdot n_{H}} \\
& =\sqrt[3]{16\left(\frac{n^{3}-3 n^{2}+3 n-1}{n^{3}-n^{2}+2 n}\right) \cdot n_{H}} \\
& =\sqrt[3]{16\left(1-\frac{2 n^{2}-n+1}{n^{3}-n^{2}+2 n}\right) \cdot n_{H}} \\
& =\sqrt[3]{16\left(1-\epsilon_{n}\right) \cdot n_{H}} \cdot
\end{aligned}
$$

Using the notation introduced in the statement of Proposition 10, we note that $\frac{2}{3}=$ $\epsilon_{3}>\epsilon_{4}>\epsilon_{5}>\cdots$ and that $\epsilon_{n} \rightarrow 0$ as $n \rightarrow \infty$. In particular, we note that given any 
$\epsilon>0$, we can choose $n$ sufficiently large so that $\epsilon_{n}<\epsilon$, implying by Proposition 10 that the connected 3-uniform hypergraph $H=H_{n}$ satisfies

$$
\sqrt[3]{16(1-\epsilon) \cdot n_{H}}<\Upsilon(H)<\sqrt[3]{16 \cdot n_{H}}
$$

\section{$5 \quad$ Closing Conjectures}

We pose the following conjecture. As observed earlier, Conjecture 11 is true for $k \in\{2,3\}$. However, we have yet to settle the conjecture for $k \geqslant 4$.

Conjecture 11. For $k \geqslant 2$, if $H$ is a connected $k$-uniform hypergraph then $\Upsilon(H) \geqslant \sqrt[k]{\frac{n_{H}}{k}}$.

Let $\mathcal{H}_{n}$ denote the class of all connected 3-uniform hypergraphs of order $n$. As observed earlier, Proposition 10 implies that for $n$ sufficiently large there exist hypergraphs $H \in \mathcal{H}_{n}$ such that

$$
\frac{\Upsilon(H)}{\sqrt[3]{n}}>\sqrt[3]{16(1-\epsilon)}
$$

for any given $\epsilon>0$. We close with the following conjecture that we have yet to settle.

Conjecture 12. $\sup _{n \rightarrow \infty}\left(\inf _{H \in \mathcal{H}_{n}} \frac{\Upsilon(H)}{\sqrt[3]{n}}\right)=\sqrt[3]{16}$.

\section{References}

[1] C. Berge, Hypergraphs - Combinatorics of Finite Sets. North-Holland, 1989.

[2] B. Bollobás and E. J. Cockayne. Graph-theoretic parameters concerning domination, independence, and irredundance. J. Graph Theory, 3:241-249, 1979.

[3] Cs. Bujtás, M. A. Henning, and Zs. Tuza. Transversals and domination in uniform hypergraphs. European J. Combin., 33:62-71, 2012.

[4] Cs. Bujtás, M. A. Henning, Zs. Tuza, and A. Yeo. Total transversals and total domination in uniform hypergraphs. Electron. J. Combin., 21(2) \#P2.24, 2014.

[5] O. Favaron. Two relations between the parameters of independence and irredundance. Discrete Math., 70:17-20, 1988.

[6] J. Gimbel and P. D. Vestergaard. Inequalities for total matchings of graphs. Ars Combin., 39:109-119, 1995.

[7] N. I. Glebov and A. V. Kostochka. On the independent domination number of graphs with given minimum degree. Discrete Math., 118:261-266, 1998.

[8] W. Goddard and M. A. Henning. Independent domination in graphs: A survey and recent results. Discrete Math., 313:839-854, 2013. 
[9] T. W. Haynes, S. T. Hedetniemi, P. J. Slater (eds). Fundamentals of Domination in Graphs, Marcel Dekker, Inc. New York, 1998.

[10] T. W. Haynes, S. T. Hedetniemi, P. J. Slater (eds). Domination in Graphs: Advanced Topics, Marcel Dekker, Inc. New York, 1998.

[11] M. A. Henning and C. Löwenstein. A characterization of the hypergraphs that achieve equality in the Chvátal-McDiarmid Theorem. Discrete Math., 323:69-75, 2014.

[12] M. A. Henning and A. Yeo. Hypergraphs with large transversal number. Discrete Math., 313:959-966, 2013.

[13] Z. Lonc and K. Warno. Minimum size transversals in uniform hypergraphs. Discrete Math., 313:2798-2815, 2013.

[14] L. Sun and J, Wang. An upper bound for the independent domination number. J. Combin. Theory, Ser. B, 76:240-246, 1999. 